\section{Fetal QRS Detection in Noninvasive Abdominal Electrocardiograms Using Principal Component Analysis and Discrete Wavelet Transforms with Signal Quality Estimation}

\author{
Mohammad Javad Mollakazemi'*® ${ }^{*}$, Farhad Asadi², Mahsa \\ Tajnesaei ${ }^{3}$ Ali Ghaffari ${ }^{4}$
}

\begin{abstract}
Background: Fetal heart rate (FHR) extracted from abdominal electrocardiogram (ECG) is a powerful non-invasive method in appropriately assessing the fetus wellbeing during pregnancy. Despite significant advances in the field of electrocardiography, the analysis of fetal ECG (FECG) signal is considered a challenging issue which is mainly due to low signal to noise ratio (SNR) of FECG.

Objective: In this study, we present an approach for accurately locating the fetal QRS complexes in non-invasive FECG.

Materials and Methods: In this experimental study, the proposed method included 4 steps. In step 1, comb notching filter was employed to pre-process the abdominal ECG (AECG). Furthermore, low frequency noises were omitted using wavelet decomposition. In next step, principal component analysis (PCA) and signal quality assessment (SQA) were used to obtain an optimal AECG reference channel for maternal R-peaks detection. In step 3, maternal ECG (MECG) was removed from mixture signal and FECG was extracted. In final step, the extracted FECG was first decomposed by discrete wavelet transforms at level 10 . Then, by employing details of levels 2, 3, 4, the new FECG signal was reconstructed in which various noises and artifacts were removed and FECG components whose frequency were close to the fetal QRS complexes remained which increased the performance of the method.
\end{abstract}

Results: For evaluation, 15 recordings of PhysioNet Noninvasive FECG database were used and the average F1 measure of $98.77 \%$ was obtained.

Conclusion: The results indicate that use of both an efficient analysis of major component of AECG along with a signal quality assessment technique has a promising performance in FECG analysis.

Citation: Mollakazemi MJ, Asadi F, Tajnesaei M, Ghaffari A. Fetal QRS Detection in Noninvasive Abdominal Electrocardiograms Using Principal Component Analysis and Discrete Wavelet Transforms with Signal Quality Estimation. J Biomed Phys Eng. 2021;11(2):197-204. doi: $10.31661 / \mathrm{jbpe}$.v0i0.397.

\section{Keywords}

Electrocardiography; Pregnancy; Fetal; Wavelet Analysis; Heart Rate; Abdomen; QRS; Fetal Monitoring; PCA; Signal Quality

\section{Introduction}

$\mathrm{F}$ Tetal electrocardiogram (FECG) is obtained by the measurement of electrical activity of the fetal heart over a period of time and is recorded by skin electrodes. FECG signal existing in the data series recorded from maternal abdomen contains vital information about
${ }^{1} \mathrm{PhD}$ Candidate, Young Researchers and Elite Club, Science and Research Branch, Islamic Azad University, Tehran, Iran

${ }^{2} \mathrm{MSc}$, Department of Mechanical Engineering K. N. Toosi University of Technology, Tehran, Iran ${ }^{3} \mathrm{MSc}$, Department of

Health Management and Economics, Tehran University of Medical Sciences, Tehran, Iran

${ }^{4} \mathrm{PhD}$, Department of Mechanical Engineering, K. N. Toosi University of Technology, Tehran, Iran

*Corresponding author: Mohammad Javad Mollakazemi

Young Researchers and Elite Club, Science and Research Branch, Islamic Azad University,

Tehran, Iran

E-mail: molakazemi. javad@yahoo.com

Received: 25 June 2015 Accepted: 10 August 2015 
the physiological state of fetal cardiovascular activity and detection of fetal cardiac arrhythmias especially when it is recorded during the last months of gestation. Generally, fetus has a faster heart rate than mother, so fundamental frequency of FECG signal is higher than maternal ECG (MECG) but its amplitude is much weaker than MECG. Consequently, FECG has small amount of signal energy relative to the maternal electrocardiogram. Due to the noninvasive nature of measurement of signal, FECG signal consists of a serious of undesired signal components. These include fetal brain activity, recording devices, movement artifacts, muscle contraction, etc. The strongest disturbance is related to maternal ECG which is 5 to 10 times bigger than FECG [1]. Furthermore, much of the duration of this signal coincides with FECG both in time and frequency domain. Therefore, locating fetal QRS complexes in its domain is a challenging task. For the extraction of FECG, many sophisticated signal processing methods have been used in literature. The approaches based on the adaptive filtering which are combined with wavelet transform [2], singular value decomposition [3], crosscorrelation method and orthogonal basis [4], independent component analysis and waveletbased techniques $[5,6]$ and so forth. These approaches can be categorized based on the principal signal processing methodologies which are adaptive filtering, linear decomposition and non-linear decomposition.

Linear decomposition methods decompose multivariate signal into different subcomponents (MECG, FECG and noises). This decomposition is done through data-driven or fixed basis functions, and the nonlinear projection and deflection approaches for subspace decomposition are applied in nonlinear decomposition method. The drawback of adaptive filtering methods is that they need a reference MECG signal to recreate the morphological shape of the MECG and the morphology of MECG is highly dependent on the electrode locations [7]. Nevertheless, among all these methods, adaptive filtering-based methods show simple algorithm structure and fast performance. Although, most approaches have the ability of feature extraction of the FECG, there are still many remaining improvements in the approaches rooted in limitations for highly spatial and temporal detection. The fusion of various approaches can improve the limitations of some approaches when they are employed alone.

The aim of this study is to present an approach in which different techniques are employed for fetal heart rate (FHR) extraction by using non-invasive abdominal ECGs. Firstly, the sample entropy of each channel was calculated based on which the channel for maternal QRS detection was selected. Then, the principal component analysis (PCA) beside discrete wavelet transforms (DWT) were employed for FECG extraction and FQRS detection. By employing the DWT for FQRS detection, the extracted FECG was decomposed and the unrequired levels were eliminated by decomposition. Then, R-peaks of the fetal were detected using the new reconstructed signal and adaptive-threshold approaches. This employment of DWT led us to know that small fetal R-peaks could be detected more accurately in contrast with other regular detection methods.

\section{Material and Methods}

\section{Materials \\ Data}

In this experimental study, physioNet Noninvasive FECG Database [8]: This fetal ECG dataset consists of 55 multichannel abdominal electrocardiogram (AECG) records, taken from a single subject between 21 and 40 weeks of pregnancy. Each record contains two thoracic channels and three or four abdominal channels. The signals are recorded at sampling frequency of $1000 \mathrm{~Hz}$ with 16-bit resolution. Moreover, a band pass filter $(0.01-100 \mathrm{~Hz})$ and a main notch filter $(50 \mathrm{~Hz})$ were applied to the data, and the fetal R-peak (FR-peak) locations 
are annotated manually. Consequently, the 13 recordings, in which the fetal QRS complexes were visible, were selected. The 13 records of PhysioNet Noninvasive fetal ECG which were used for evaluation are: ecgca154, 192, 323, 368, 444, 597, 649, 746, 748, 811, 826, 848, and 906. In some selected recordings, fetal beats could be observed only after denoising. The second minute of 13 selected recordings was extracted and used for evaluation.

\section{Performance Evaluation}

Different measurement approaches for evaluation of FR-peak detection were used. The first two indexes were sensitivity, Se, positive diagnostic value, PDV, as defined in ANSI/ AAMI EC57 standard [9]. Furthermore, equations (1) and (2) describe two measures that were used to summarize the accuracy of the approach, for instance, true positive (TP) in comparison to false positive (FP) and false negative (FN): accuracy (ACC) as in [10] and the F1-measure [11]:

$$
\mathrm{Acc}=\frac{\mathrm{TP}}{\mathrm{TP}+\mathrm{FN}+\mathrm{FP}} \times 100
$$

$$
F_{1}=2 \frac{\mathrm{PDV} \cdot \mathrm{Se}}{\mathrm{PDV}+\mathrm{Se}} \times 100=\frac{2 T P}{2 T P+F P+F N} \times 100
$$

Noise Component Identification for Better Information Extraction of MECG and Signal Quality for Components of PCA Analysis

Statistical decomposition algorithms such as principal component analysis (PCA) have been applied for multi-channel ECG extensive analysis and noninvasive FECG extraction $[12,13]$. However, there are many concerns such as noise-sensitivity of the extracted components of this algorithm. In [14], it is shown that frequency spectrum of each noise and MECG overlaps with FECG, and hence PCA that is based on obtaining statistically uncorrelated components, do not necessarily show independent physiological sources accurately.

These noises are typically very non-station- ary in time and colored in spectrum. In other words, they have long-term correlations. However, one of the limitations of PCA is that MECG, FECG and source of the noises are assumed to be a linear and stationary mixture, and also this method is regarded as spatial filter rather than temporal filters. Thereby, for improving the quality of components of PCA algorithm and quality of signal, sample entropy is used for quantifying unpredictability and regularity of noises in signal [15]. When raw AECG signal is recorded, unexpected noises affect the extracted information from FECG. Sample entropy is used for determination of noise components and signal quality assessment (SQA) in signal. In preprocessing section, channel with the smallest value of sample entropy is chosen and hence this methodology makes improvement and assessment of the first principal component.

\section{Methods}

In our proposed algorithm, different algorithms are incorporated consecutively. In an overall view, the proposed algorithm has two complementary steps. In the first step, MECG components are separated from the composite signal. This separation and removing is implemented in the best channel selected based on the estimated sample entropy. Then, the dominant components of FECG are extracted, and the QRS complexes are detected by multi-resolution analysis of extracted FECG. The flowchart of the algorithm explaining the processing steps of the approach is shown in Figure 1.

Preprocessing

In the preprocessing step, baseline wandering noise is removed by applying the discrete wavelet transform. Then, the signal is processed further to remove power line interference by using comb notching filter. Afterwards, two different criteria are considered where PCA and entropy quantification are implemented in order to determine the optimal channel for maternal heart beats. These approaches are considered for rating the chan- 


\begin{tabular}{|c|c|c|c|}
\hline Preprocessing & $\begin{array}{c}\text { Maternal R-peak } \\
\text { detection }\end{array}$ & $\begin{array}{l}\text { MECG } \\
\text { Elimination }\end{array}$ & $\begin{array}{l}\text { Fetal R-peak } \\
\text { detection }\end{array}$ \\
\hline $\begin{array}{l}\text { - Filtering } \\
\text { - PCA Analysis } \\
\text {-Trend removal by wavelet } \\
\text { decomposition } \\
\text {-band pass filter }\end{array}$ & $\begin{array}{l}\text { - Quality assessment } \\
\text { - Determine optimal reference } \\
\text { channel } \\
\text { - Candidate MQRS detection } \\
\text { - MQRS candidates finalization }\end{array}$ & $\begin{array}{l}\text { - MECG construction } \\
\text { - FECG extraction by } \\
\text { removing MECG from } \\
\text { AECG signal }\end{array}$ & $\begin{array}{l}\text { - FECG decomposition by DWT } \\
\text { - FECG reconstruction } \\
\text { - PCA to enhance FECG } \\
\text { - Selection of the best detected } \\
\text { FQRS annotations }\end{array}$ \\
\hline
\end{tabular}

Figure 1: Schematic flowchart of the processing stages (left to right).

nels. For instance, under conditions when the signal has noise with high frequency, sample entropy has a bigger value and this rating value is chosen to value of 1.5 , and regarding channels with less noise, sample entropy has smaller values. Then, the chosen channel is processed by the PCA approach which makes assessment and improvement for PCA results. So, this criterion is employed and finally the best channel with minimum sample entropy is chosen for maternal $\mathrm{R}$ peak detection.

Maternal R-peak Detection

In this section, the candidate maternal Rpeaks from the channel whose score is the highest, are detected by using Pan and Tompkins, an adaptive QRS detector [16]. Next, actual R-peaks of MQRS from the channel with the highest signal quality are considered as finalized detections of maternal R-peak candidate locations.

\section{MECG Cancellation}

In summary, in this step for channels having features of high quality, MECG morphology was constructed according to the real length of each period and then by joining them, pure FECG signal is extracted when the related MECG components were removed in the channels.

\section{Fetal R-peak Detection}

In the last step, the extracted FECG signal was first decomposed by discrete wavelet transforms (with function of 'db6' in level 10). Then, the decomposed signal was reconstructed using detailed signal of levels 2, 3 and 4, and fetal R-peaks were then detected through the energy of the reconstructed signal. Finally, the fetal R-peaks of the channels whose variance of RR-interval was smallest were chosen as the final fetal R-peaks.

\section{Results}

For evaluating the proposed approach, it was applied on 15 recordings of PhysioNet noninvasive fECG database. Briefly explaining, the second minutes of the records are extracted and used for evaluation and the records were annotated manually. Consequently, the recordings in which FQRS complexes were visible (in some cases after preprocessing) were selected for evaluation. The results are shown in Table 1.

\section{Discussion}

In this paper, since noisy channels will adversely affect PCA results, the sample entropy was used to evaluate the signal quality and to exclude the channels with high amount of noises. The final fetal R-peaks were reported by comparing the variance of series of RR-interval of the fetal R-peaks time series for each FECG channel. The series with the smallest variance value were assumed that the established order of the detected FQRS complexes was better than the other channels with bigger variance values. For instance, as in Figure 2, the first FECG channel contained more amounts of various noises. The detected FQRS complexes were not on the specific discipline. Consequently, the value of the fetal RR-intervals was bigger than other two chan- 
Table 1: The results of the proposed method using the PhysioNet noninvasive Fetal electrocardiogram (FECG) database.

\begin{tabular}{ccccc} 
Record Name & Se (\%) & PDV (\%) & Acc (\%) & F1 (\%) \\
\hline ecgca154 & 98.67 & 100 & 98.67 & 99.33 \\
\hline ecgca192 & 100 & 100 & 100 & 100 \\
\hline ecgca323 & 100 & 100 & 100 & 100 \\
\hline ecgca368 & 97.22 & 99.29 & 96.55 & 98.25 \\
\hline ecgca444 & 94.30 & 99.33 & 93.71 & 96.75 \\
\hline ecgca597 & 94.37 & 99.34 & 93.79 & 96.80 \\
\hline ecgca649 & 99.36 & 99.36 & 98.73 & 99.36 \\
\hline ecgca746 & 99.32 & 100 & 98.31 & 99.66 \\
\hline ecgca748 & 98.72 & 99.35 & 98.09 & 99.03 \\
\hline ecgca811 & 100 & 100 & 100 & 100 \\
\hline ecgca826 & 97.42 & 98.01 & 95.56 & 97.73 \\
\hline ecgca848 & 96.85 & 99.35 & 96.25 & 98.09 \\
\hline ecgca906 & 97.94 & 100 & 97.94 & 98.96 \\
\hline Total & 98.01 & 99.54 & 97.51 & 98.77
\end{tabular}

Se: Sensitivity, PDV: Positive diagnostic value, ACC: Accuracy

nels. The proposed method performs well even if the recordings with one or two AECG channels were seriously polluted by noises. On the other hand, in the recordings which the FECG signal are really weak in a way that FQRS complexes could not be observed easily, the exhausted results of the proposed technique will reduce. A comparison of the performance of the proposed approach with other studies is illustrated in Table 2.

\section{Conclusion}

In this study, an approach was presented to detect FQRS complexes from non-invasive abdominal ECG. The exhausted results were calculated using extracted one-minute AECG signal from 13 recordings of PhysioNet Noninvasive FECG Database. By developing one multi-step approach, both fetal and maternal QRS complexes are located from AECG recording with appropriate accuracy in comparison to other references. In this research, by using signal quality index and taking the sample entropy of each channel, the unpredictability and regularity of noise components are discriminated and then PCA approach is applied to each channel. These steps can enhance the ability of the algorithm for choosing the optimal AECG reference channel for maternal R-peak detection. Moreover, methodologies such as multi resolution approach for decomposition of the extracted FECG signal and estimation of the variance of the detected fetal RR-intervals have enhanced the performance of the algorithm for detecting the components of FECG whose frequency are close to the fetal QRS frequency and have improved the accuracy of the final reported FR-peaks locations and consequently, the final measured FHR demonstrates higher accuracy.

\section{Conflict of Interest}

None

\section{References}

1. Sameni R, Clifford GD. A Review of Fetal ECG Signal Processing; Issues and Promising Directions. Open Pacing Electrophysiol Ther J. 2010;3:420. doi: $10.2174 / 1876536 \times 01003010004$. 
MQRS Detection Results, channel 1

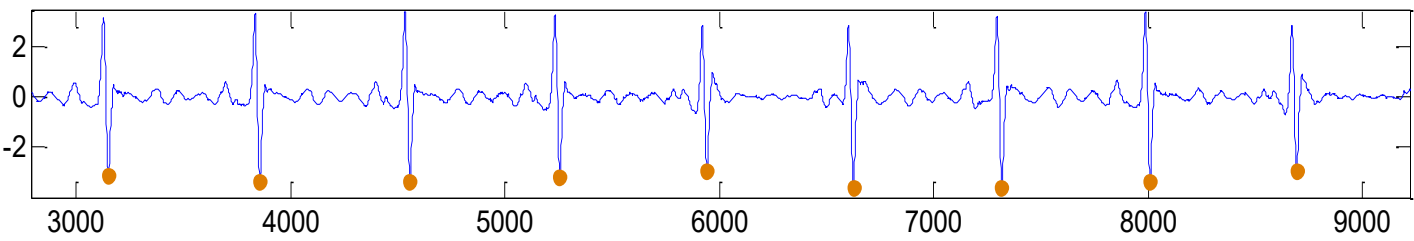

channel 2

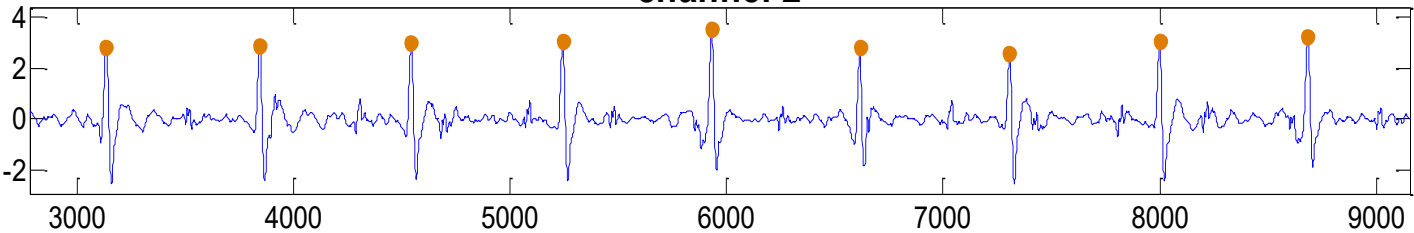

channel 3

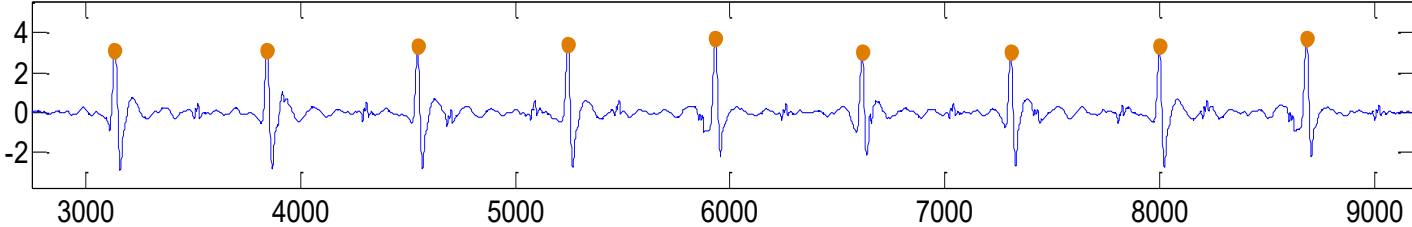

FECG, channel 1

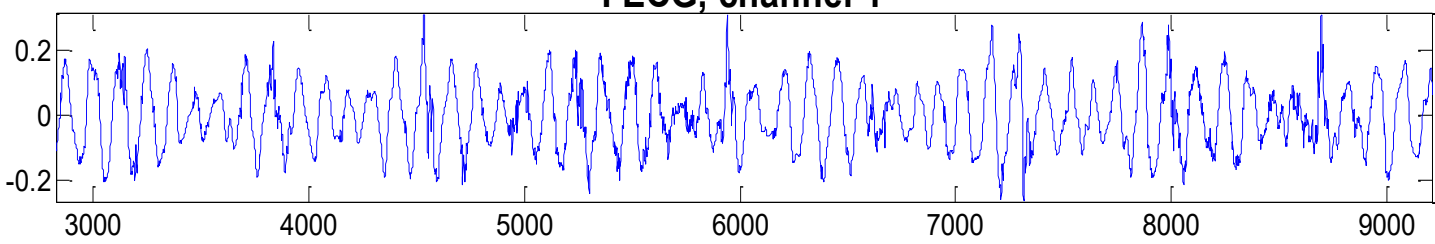

FECG, channel 2 (Optimal Channel)

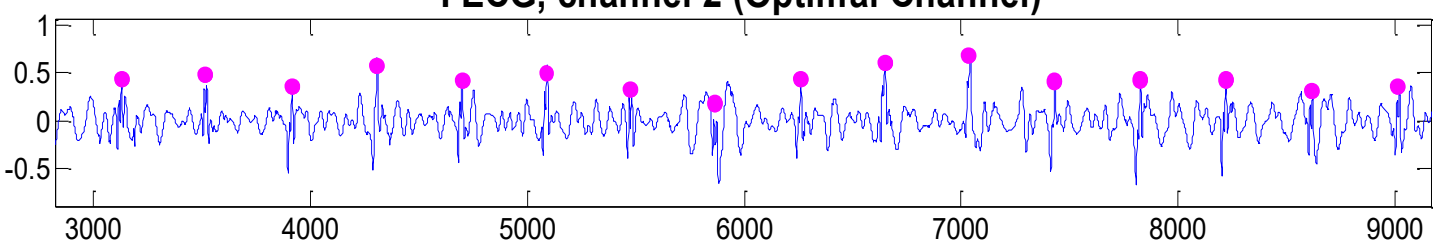

FECG, channel 3

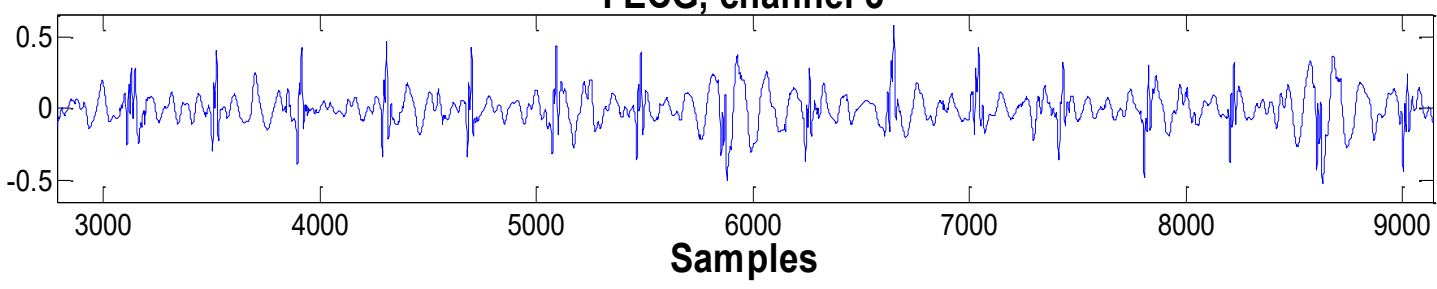

Figure 2: (Up to down) -The first three channels: The maternal QRS detection results on original abdominal electrocardiogram (AECG) -The second three channels: The extracted Fetal electrocardiogram (FECG) and the detected fetal QRS complexes in optimal FECG channel

PubMed PMID: 21614148. PubMed PMCID: 3100207.

2. Wu S, Shen Y, Zhou Z, Lin L, Zeng Y, Gao $X$. Research of fetal ECG extraction using wavelet analysis and adaptive filtering. Com- put Biol Med. 2013;43:1622-7. doi: 10.1016/j. compbiomed.2013.07.028. PubMed PMID: 24034754.

3. Hasan MA, Reaz MB, Ibrahimy MI, Hussain MS, Uddin J. Detection and Processing Tech- 
Fetal QRS Detection by PCA, DWT and SQE

Table 2: Comparison of this study with existing works for fetal heart rate (FHR) measurement.

\begin{tabular}{lcc}
\multicolumn{1}{c}{ Author } & Database & Acc (\%) \\
\hline Ibrahimy et al., 2003 [17] & 5 records-1a & 89 \\
\hline Karvounis et al., 2004 [18] & 15 records-3 & 98.94 \\
\hline Martens et al., 2007 [13] & 20 records-13 & 85 \\
\hline Karvounis et al., 2009 [19] & 13 records-3 & 95.45 \\
\hline Hasan et al, 2012 [20] & 10 records & $93.75 b$ \\
\hline Behar et al., 2014 [21] & 11 records-28 & $90.2 \mathrm{c}$ \\
\hline Ghaffari et al., 2015 [5] & 69 records-4 & 83.62 \\
\cline { 2 - 3 } & 26 records-3 or 4 & 98.21 \\
\hline This study & 13 records-3 or 4 & 97.51 \\
ACC: Accuracy & &
\end{tabular}

niques of FECG Signal for Fetal Monitoring. Biol Proced Online. 2009;11:263-95. doi: 10.1007/ s12575-009-9006-z. PubMed PMID: 19495912. PubMed PMCID: 3055800.

4. Christov I, Simova I, Abacherli R. Extraction of the fetal ECG in noninvasive recordings by signal decompositions. Physiol Meas. 2014;35:171321. doi: 10.1088/0967-3334/35/8/1713. PubMed PMID: 25070127.

5. Ghaffari A, Mollakazemi MJ, Atyabi SA, Niknazar M. Robust fetal QRS detection from noninvasive abdominal electrocardiogram based on channel selection and simultaneous multichannel processing. Australas Phys Eng Sci Med. 2015;38:581-92. doi: 10.1007/s13246-0150381-2. PubMed PMID: 26462679.

6. Ghaffari A, Atyabi S, Mollakazemi MJ, Niknazar M, Niknami M, Soleimani A, editors. PhysioNet/ CinC Challenge 2013: a novel noninvasive technique to recognize fetal QRS complexes from noninvasive fetal electrocardiogram signals. Computing in Cardiology Conference; Zaragoza, Spain: IEEE; 2013.

7. Liu C, Li P, Di Maria C, Zhao L, Zhang H, Chen Z. A multi-step method with signal quality assessment and fine-tuning procedure to locate maternal and fetal QRS complexes from abdominal ECG recordings. Physiol Meas. 2014;35:166583. doi: $10.1088 / 0967-3334 / 35 / 8 / 1665$. PubMed PMID: 25069817.

8. Goldberger AL, Amaral LA, Glass L, Hausdorff JM, Ivanov PC, Mark RG, et al. PhysioBank,
PhysioToolkit, and PhysioNet: components of a new research resource for complex physiologic signals. Circulation. 2000;101:E215-20. doi: 10.1161/01.CIR.101.23.e215. PubMed PMID: 10851218.

9. American National Standard. ANSI/AAMI/ISO EC57 2012: Testing and reporting performance results of cardiac rhythm and ST segment. Available from: http://webstore.ansi.org/Previews/PREVIEW_ANSI+AAMI+EC57-2012.pdf.

10. Karvounis EC, Tsipouras MG, Fotiadis DI, Naka KK. An automated methodology for fetal heart rate extraction from the abdominal electrocardiogram. IEEE Trans Inf Technol Biomed. 2007;11:628-38. doi: 10.1109/ TITB.2006.888698. PubMed PMID: 18046938.

11. Behar J, Oster J, Clifford GD, editors. Non-invasive FECG extraction from a set of abdominal sensors. Computing in Cardiology Conference; Zaragoza, Spain: IEEE; 2013.

12. Di Maria C, Duan W, Bojarnejad M, Pan F, King $\mathrm{S}$, Zheng D, Murray A, Langley Ph, editors. An algorithm for the analysis of fetal ECGs from 4-channel non-invasive abdominal recordings. Computing in Cardiology Conference; Zaragoza, Spain: IEEE; 2013.

13. Martens SM, Rabotti C, Mischi M, Sluijter RJ. A robust fetal ECG detection method for abdominal recordings. Physiol Meas. 2007;28:373-88. doi: $10.1088 / 0967-3334 / 28 / 4 / 004$. PubMed PMID: 17395993.

14. Tompkins WJ. Biomedical digital signal processing: C-language Examples and Laboratory Experiments for the IBM PC. United States: Prentice-Hall, Inc; 1993.

15. Costa M, Goldberger AL, Peng CK. Multiscale entropy analysis of biological signals. Phys Rev E Stat Nonlin Soft Matter Phys. 2005;71:021906. doi: 10.1103/PhysRevE.71.021906. PubMed PMID: 15783351.

16. Pan J, Tompkins WJ. A real-time QRS detection algorithm. IEEE Transactions on Biomedical Engineering. 1985;BME-32(3):230-6. doi: 10.1109/TBME.1985.325532.

17. Ibrahimy MI, Ahmed F, Mohd Ali MA, Zahedi E. Real-time signal processing for fetal heart rate monitoring. IEEE Trans Biomed Eng. 2003;50:258-62. doi: 10.1109/ TBME.2002.807642. PubMed PMID: 12665042.

18. Karvounis E, Papaloukas C, Fotiadis DI, Michal- 
is $L$, et al. Fetal heart rate extraction from composite maternal ECG using complex continuous wavelet transform. Computers in Cardiology. 2004;31:737-40.

19. Karvounis EC, Tsipouras MG, Fotiadis DI. Detection of fetal heart rate through 3-D phase space analysis from multivariate abdominal recordings. IEEE Trans Biomed Eng. 2009;56:1394406. doi: 10.1109/TBME.2009.2014691. PubMed PMID: 19228552.
20. Hasan M, Reaz M. Hardware prototyping of neural network based fetal electrocardiogram extraction. Measurement Science Review. 2012;12:52-5. doi: 10.2478/v10048-012-00078.

21. Behar J, Johnson A, Clifford GD, Oster J. A comparison of single channel fetal ECG extraction methods. Ann Biomed Eng. 2014;42:134053. doi: 10.1007/s10439-014-0993-9. PubMed PMID: 24604619. 